\title{
Instability of Nonlinear Bound States
}

\author{
Jalal Shatah $^{1}$ and Walter Strauss ${ }^{2}$ \\ 1 Courant Institute, New York, New York 10012, USA \\ 2 Department of Mathematics, Brown University, Providence, Rhode Island 02912, USA
}

\begin{abstract}
We establish a sharp instability theorem for the bound states of lowest energy of the nonlinear Klein-Gordon equation, $u_{t t}-\Delta u+f(u)=0$, and the nonlinear Schrödinger equation, $-i u_{t}-\Delta u+f(u)=0$.
\end{abstract}

\section{Introduction}

In this paper we prove a sharp instability theorem for the bound states of lowest energy of the nonlinear Klein-Gordon equation

(NLKG)

$$
u_{t t}-\Delta u+f(u)=0
$$

and the nonlinear Schrödinger equation

$$
-i u_{t}-\Delta u+f(u)=0 .
$$

By a bound state we mean a solution of the form

$$
u(x, t)=e^{i \omega t} \phi_{\omega}(x)
$$

with $\omega$ a real parameter and $\phi_{\omega}(x)$ suitably vanishing as $|x| \rightarrow \infty$. The nonlinearity $f$ is very general: it satisfies conditions which are sufficient and are almost necessary for the existence of such bound states. Each of these systems is distinguished by a pair of invariants, energy $E$ and charge $Q$. Energy comes from the time invariance and charge from the gauge invariance. It is the gauge invariance which makes the problem interesting. It means that $\phi_{\omega}(x) \exp i(\omega t+\theta)$ is a bound state for any constant $\theta$. Our original proof of instability for NLKG also made use of the broken dilation invariance. However, this method gave a sharp result only in the case of a pure power nonlinearity $f(u)=u-|u|^{p-1} u, p>1$ (cf. Sect. 4). The general case requires a more abstract method.

In general the states are stable for some $\omega$ and unstable for other $\omega$. Stability 
of a bound state means that any initial datum sufficiently near this state gives rise to a solution which exists and remains near the state for all subsequent times. The sharp instability condition is

$$
\frac{d}{d \omega} Q\left(\phi_{\omega}\right)>0
$$

Another way to express this is that the action $L\left(\phi_{\omega}\right)$ be a concave function of $\omega$. With the opposite (strict) condition we have stability. For example, take the pure power nonlinearity mentioned above. For NLS stability is characterized by the condition $p<1+4 / n$. But for NLKG the condition depends on the frequency: $p<1+4 / n$ and $|\omega|>\omega_{c}$ for a certain critical frequency $\omega_{c}$. For general nonlinearities, NLS is always more stable than NLKG. Another feature which distinguishes NLS is the positivity of the charge, which helps to simplify the analysis considerably.

Blow up theorems have been proved in [9,5 and 2]. The case of $\omega=0$ but arbitrary $f$, when the solution might exist for all time (as well as some cases with $\omega \neq 0$ ), was treated in [11]. In [6] was considered a similar problem with dissipation. The stability theorem was proved in [10] for NLKG. For NLS in the pure power case the stability result was proved in [4]. The linearized operator for NLS was analysed in [15]. The results of the present paper were announced in part in [14], in the A.M.S. meeting in Berkeley in August, 1983 and in AMS Abstracts 3 (1982), p. $397 ; 4$ (1983), p. 498; 5 (1984), p. 399. In a forthcoming paper we shall generalize these results to systems invariant under a more general gauge group.

The possibility of stable bound states for $\omega \neq 0$ was first suggested in [1]. Subsequent numerical investigations as well as heuristic arguments based on physical intuition led to a formal understanding of the problem $([7,8])$. The idea is to graph the energy $E\left(\phi_{\omega}\right)$ against the charge $Q\left(\phi_{\omega}\right)$. If two frequencies have the same charge, then the lower energy state is stable and the higher one unstable. This idea is borne out by our analysis.

In Sect. 1 we construct the ground state $\phi_{\omega}$ and discuss the behavior of the energy and charge near $\phi_{\omega}$ (for NLKG). In Sect. 2 we construct a certain local functional $P$ which measures the deviation of the energy from its value at $\phi_{\omega}$. In Sect. 3 we use $P$ to prove the instability for NLKG. Section 4 contains examples and comments. In Sect. 5 we treat the Schrödinger equation by the identical method. In Sect. 6 we make some technical comments which extend the scope of our results.

\section{Standing Waves}

In this section we discuss the existence of standing waves and their dependence on the frequency. The equation

$$
\left(\partial_{t}^{2}-\Delta\right) u+f(u)=0 \quad\left(x \in \mathbb{R}^{n}, n \geqq 2\right),
$$

where

$$
f(0)=0, \quad f^{\prime}(0)=1, \quad f\left(e^{i \theta} u\right)=e^{i \theta} f(u) \text { for } \theta \in \mathbb{R}
$$


has the standing wave solution $u(x, t)=\phi(x) \exp (i \omega t)$, where $\omega$ is real, provided

$$
-\Delta \phi+f(\phi)-\omega^{2} \phi=0 .
$$

We assume that $f$ is a $C^{1}$ function, real for real arguments, which satisfies

$$
\begin{gathered}
F(\eta)-\frac{1}{2} \omega^{2} \eta^{2}<0 \text { for some } \eta \text { and some } \omega^{2}<1, \\
\left|f^{\prime}(s)\right|=o\left(|s|^{4 /(n-2)}\right) \text { as }|s| \rightarrow \infty,
\end{gathered}
$$

where $F^{\prime}=f, F(0)=0$. Conditions $(H 1)$ and $(H 2)$ are sufficient for (2) to have non-trivial solutions which vanish at infinity $[13,3]$. Condition $(H 2)$ can be weakened (see Sect. 6 below). If $n=2$, the exponent in (H2) should be replaced by an arbitrary power.

The function space in which we shall work is $X=H_{r}^{1} \oplus L_{r}^{2}$, the space of pairs of complex-valued functions of $r=|x|$ which belong to $H^{1}\left(\mathbb{R}^{n}\right) \oplus L^{2}\left(\mathbb{R}^{n}\right)$. An element of $X$ is denoted by $\vec{u}=\left[u_{1}, u_{2}\right]$. We use the square bracket to distinguish a pair from an inner product. We regard $X$ as a real Hilbert space with the inner product

$$
(\vec{u}, \vec{v})=\operatorname{Re} \int\left(\nabla u_{1} \nabla \bar{v}_{1}+u_{1} \bar{v}_{1}+u_{2} \bar{v}_{2}\right) d x .
$$

The real dual space of $X$ will be denoted by $X^{*}$. For $\vec{f} \in X^{*}$ and $\vec{u} \in X,\langle\vec{f}, \vec{u}\rangle$ denotes the value of $\vec{f}$ at $\vec{u}$. We define $\overrightarrow{i f}$ by $\langle\overrightarrow{i f}, \vec{u}\rangle=-\langle\vec{f}, i \vec{u}\rangle$. We define an identification operator $I: X \rightarrow X^{*}$ as follows: $f=I(\vec{u}) \in X^{*}$,

$$
\langle\vec{f}, \vec{v}\rangle=\operatorname{Re} \int\left(u_{1} \bar{v}_{1}+u_{2} \bar{v}_{2}\right) d x
$$

We define a map $J: X \rightarrow X^{*}$

$$
J=I\left(\begin{array}{rr}
0 & -1 \\
1 & 0
\end{array}\right)
$$

acting on $\vec{u}$ as a column vector. That is,

$$
\langle J(\vec{u}), \vec{v}\rangle=\operatorname{Re} \int\left(-u_{2} \bar{v}_{1}+u_{1} \bar{v}_{2}\right) d x .
$$

Note that $J^{*}=-J$. Multiplication by $i=\sqrt{-1}$ commutes with $I$ and with $J$.

If we define on $X$ the energy functional

$$
E(\vec{u})=\int\left(\frac{1}{2}\left|u_{2}\right|^{2}+\frac{1}{2}\left|\nabla u_{1}\right|^{2}+F\left(u_{1}\right)\right) d x,
$$

then the nonlinear wave Eq. (1) can be written as the Hamiltonian system

$$
J \frac{d \vec{u}}{d t}=E^{\prime}(\vec{u})
$$

Furthermore, Eq. (2) can be written as

$$
\omega(J i \vec{\phi})=E^{\prime}(\vec{\phi}), \quad \text { where } \vec{\phi}=[\phi, i \omega \phi] .
$$

Nontrivial solutions of (2), or equivalently (8), are obtained using variational techniques. For this reason we define the following additional functionals. 
Definition 1. For $\vec{u} \in X$,

$$
\begin{aligned}
& Q(\vec{u})=\frac{1}{2}\langle J \vec{u}, i \vec{u}\rangle=\operatorname{Im} \int \bar{u}_{1} u_{2} d x, \quad \text { (charge) } \\
& L(\vec{u})=\int\left(-\frac{1}{2}\left|u_{2}\right|^{2}+\frac{1}{2}\left|\nabla u_{1}\right|^{2}+F\left(u_{1}\right)\right) d x, \quad \text { (Lagrangian) } \\
& K(\vec{u})=\int\left(-\frac{1}{2}\left|u_{2}\right|^{2}+\left(\frac{1}{2}-\frac{1}{n}\right)\left|\nabla u_{1}\right|^{2}+F\left(u_{1}\right)\right) d x .
\end{aligned}
$$

By the growth restriction $(H 2)$ on $f$ and the Sobolev inequality, all of these functionals are $C^{2}$ on $X$. Note that $Q^{\prime}(\vec{u})=J i \vec{u}$. Hence the solutions of (8) are critical points of $E(\vec{u})$ restricted to the manifold $Q(\vec{u})=$ constant. In fact,

$$
E^{\prime}(\vec{\phi})=\omega Q^{\prime}(\vec{\phi})
$$

Let $\omega^{*}<|\omega|<1$ be the interval of $\omega$ so that $(H 1)$ holds. Let $-1<\omega<1$ in case $F$ is itself somewhere negative.

Theorem 2. Let $d(\omega)=\inf \left\{1 / n \int|\nabla u|^{2} d x: 0 \neq u \in H_{r}^{1}, K(u, i \omega u) \leqq 0\right\}$.

Then a) the infimum is achieved at some $u=\phi>0$,

b) $K(\phi, i \omega \phi)=0$,

c) $\vec{\phi}=[\phi, i \omega \phi]$ satisfies $(8)$,

d) $d(\omega)=\inf \left\{L(u, i \omega u): 0 \neq u \in H_{r}^{1}, K(u, i \omega u)=0\right\}$,

e) $d(\omega)=\inf \left\{L(u, i \omega u): \int|\nabla u|^{2} d x=\int|\nabla \phi|^{2} d x\right\}$.

Proof. This theorem may be found in [11] for $n \geqq 3$, so we merely sketch the proof here. Write $K(u)=K(u, i \omega u)$ and $L(u)=L(u, i \omega u)$. Firstly, note that $K(u) \geqq c\|u\|^{2}$ for $\|u\|$ small where $c>0$. Hence $\left\{u: 0 \neq u \in H^{1}, K(u) \leqq 0\right\}$ is bounded away from 0 in $X$. Secondly, take a minimizing sequence $\left(u_{j}\right)$ for $d(\omega)$. By $(H 2)$, $0 \geqq K\left(u_{j}\right) \geqq c\left\|u_{j}\right\|^{2}-\left\|u_{j}\right\|^{\alpha}$, where $\alpha>2$. This implies that $\left(u_{j}\right)$ is a bounded sequence in $H_{r}^{1}$. The compact embedding $H_{r}^{1} \rightarrow L_{r}^{p}$ for $2<p<2+4 /(n-2)$ (see [13]) and condition (H2) imply that the functional $K$ is lower semi-continuous. So there is a subsequence converging to some $\phi \in H_{r}^{1}$. Also $K(\phi) \leqq \underline{\lim } K\left(u_{j}\right) \leqq 0$ and $1 / n \int|\nabla \phi|^{2} \leqq d(\omega)$. Since $K$ is bounded away from zero, $\phi \neq 0$ and therefore the convergence is strong. The positivity of $\phi$ follows from the maximum principle and the minimization. This proves a).

Thirdly, consider the curve $\lambda \rightarrow \phi(\cdot / \lambda) \in H_{r}^{1}$. Since at $\lambda=1$ we have a minimum, $K(\phi)=0$, proving b). Fourthly, note that $L(u)=1 / n \int|\nabla u|^{2}+K(u)$. Thus a) and b) imply d). The Euler-Lagrange equation for d) can be written in the form (2) or equivalently (8). Fifthly, if

$$
\int|\nabla v|^{2} d x=\int|\nabla \phi|^{2} d x \text { with } v \in H_{r}^{1},
$$

then $K(v) \geqq 0$ from step one. Therefore

$$
L(v)=\frac{1}{n} \int|\nabla v|^{2}+K(v) \geqq \frac{1}{n} \int|\nabla \phi|^{2} d x=L(\phi) .
$$

This completes the sketch of the proof if $n \geqq 3$. For $n=2$, see $[16,17]$.

Uniqueness of the solution $\phi$ is not known in general. Nevertheless we assume that there is a choice $\phi=\phi_{\omega}$ which achieves the infimum of Theorem 2 such 
that $\omega \rightarrow \phi_{\omega}$ is a $C^{2}$ mapping from the interval $\left(\omega^{*}, 1\right)$ into $H_{r}^{1}$. This assumption is not necessary (see Sect. 6) but it simplifies the rest of the presentation. Now we have a $C^{2}$ curve $\vec{\phi}_{\omega}=\left[\phi_{\omega}, i \omega \phi_{\omega}\right]$ in $X$ which is a nontrivial solution of $E^{\prime}\left(\vec{\phi}_{\omega}\right)=$ $\omega J i \vec{\phi}_{\omega}$. Furthermore,

$$
d(\omega)=\frac{1}{n} \int\left|\nabla \phi_{\omega}\right|^{2} d x=L\left(\vec{\phi}_{\omega}\right) .
$$

Lemma 3. For convenience we take $\omega>0$. Then $d(\omega)$ is a positive decreasing function of $\omega$ and $d^{\prime}(\omega)=-\omega \int\left|\phi_{\omega}\right|^{2} d x=-Q\left(\bar{\phi}_{\omega}\right)$.

Proof. Since $d(\omega)=L\left(\vec{\phi}_{\omega}\right)=E\left(\vec{\phi}_{\omega}\right)-\omega Q\left(\vec{\phi}_{\omega}\right)$,

$$
d^{\prime}(\omega)=\left\langle E^{\prime}\left(\vec{\phi}_{\omega}\right)-\omega Q^{\prime}\left(\vec{\phi}_{\omega}\right), \quad d \vec{\phi}_{\omega} / d \omega\right\rangle-Q\left(\vec{\phi}_{\omega}\right) .
$$

But the first term vanishes by (9). Therefore

$$
d^{\prime}(\omega)=-Q\left(\vec{\phi}_{\omega}\right) .
$$

Lemma 4. Fix $\omega=\omega_{0}$ and let $\vec{\phi}_{0}=\vec{\phi}_{\omega_{0}}$. Then for any $C^{2}$ curve $\vec{u}(\lambda)$ such that $\vec{u}(0)=\vec{\phi}_{0}$ and $Q(\vec{u}(\lambda))=Q\left(\vec{\phi}_{0}\right)$, we have

$$
\frac{d^{2}}{d \lambda^{2}} E(\vec{u}(\lambda)) /_{\lambda=0}=\left\langle\left(E^{\prime \prime}\left(\vec{\phi}_{0}\right)-\omega_{0} Q^{\prime \prime}\left(\vec{\phi}_{0}\right)\right) \vec{y}_{0}, \vec{y}_{0}\right\rangle
$$

where

$$
\vec{y}_{0}=\vec{u}^{\prime}(0)
$$

Proof. Differentiating $E$ along the curve $\vec{u}(\lambda)$,

$$
\begin{gathered}
\frac{d}{d \lambda} E(\vec{u}(\lambda))=\left\langle E^{\prime}(\vec{u}(\lambda)), d \vec{u} / d \lambda\right\rangle, \\
\frac{d^{2}}{d \lambda^{2}} E(\vec{u}(\lambda))=\left\langle E^{\prime \prime}(\vec{u}) \vec{u}^{\prime}, \vec{u}^{\prime}\right\rangle+\left\langle E^{\prime}(\vec{u}), \vec{u}^{\prime \prime}\right\rangle .
\end{gathered}
$$

Since $Q$ is constant on the curve,

$$
\frac{d^{2}}{d \lambda^{2}} Q(\vec{u}(\lambda))=\left\langle Q^{\prime \prime}(\vec{u}) \vec{u}^{\prime}, \vec{u}^{\prime}\right\rangle+\left\langle Q^{\prime}(\vec{u}), \vec{u}^{\prime \prime}\right\rangle=0 .
$$

Subtracting $\omega_{0}$ times (13) from (12) and evaluating the result at $\lambda=0$, we obtain

$$
\left\langle\left(E^{\prime \prime}\left(\vec{\phi}_{0}\right)-\omega_{0} Q^{\prime \prime}\left(\vec{\phi}_{0}\right)\right) \vec{y}_{0}, \vec{y}_{0}\right\rangle+\left\langle E^{\prime}\left(\vec{\phi}_{0}\right)-\omega_{0} Q^{\prime}\left(\vec{\phi}_{0}\right), \vec{u}^{\prime \prime}\right\rangle .
$$

The last term vanishes by (9).

Theorem 5. Consider a frequency $\omega_{0}$ and the corresponding solution $\vec{\phi}_{0}=\phi_{\omega_{0}}$. Then $d(\omega)$ is convex at $\omega_{0}$ if and only if the functional E restricted to the manifold $M_{0}$

$$
M_{0} \equiv\left\{\vec{u} \in X \mid Q(\vec{u})=Q\left(\vec{\phi}_{0}\right)\right\}
$$

has a local minimum at $\vec{\phi}_{0}$. 
Proof. For the necessity we note that

$$
\begin{aligned}
E(\vec{u})= & \int\left\{\frac{1}{2}\left|u_{2}\right|^{2}+\frac{1}{2}\left|\nabla u_{1}\right|^{2}+F\left(u_{1}\right)\right\} d x \\
= & \int \frac{1}{2}\left|u_{2}-i \omega u_{1}\right|^{2} d x+\int\left\{-\frac{1}{2} \omega^{2}\left|u_{1}\right|^{2}+\frac{1}{2}\left|\nabla u_{1}\right|^{2}+F\left(u_{1}\right)\right\} d x \\
& +\omega \operatorname{Im} \int u_{1} \bar{u}_{2} ; \text { that is } \\
E(\vec{u}) \geqq & L\left(u_{1}, i \omega u_{1}\right)+\omega Q(\vec{u}) .
\end{aligned}
$$

For any $\vec{u} \in M_{0}$ in a small neighborhood of $\vec{\phi}_{0}$, we can find an $\omega$ so that $\int\left|\nabla u_{1}\right|^{2} d x=\int\left|\nabla \phi_{\omega}\right|^{2} d x$ because $d^{\prime}(\omega)=-\omega \int\left|\phi_{\omega}\right|^{2} \neq 0$. By Theorem 2(e), (15) becomes

$$
E(\vec{u}) \geqq d(\omega)+\omega Q\left(\vec{\phi}_{0}\right)=d(\omega)-\omega d^{\prime}\left(\omega_{0}\right) .
$$

If $d(\omega)$ is convex, (16) implies

$$
E(\vec{u}) \geqq d\left(\omega_{0}\right)-\omega_{0} d^{\prime}\left(\omega_{0}\right)=E\left(\vec{\phi}_{0}\right)
$$

for $u \in M_{0}$ in a small neighborhood of $\vec{\phi}_{0}$. Therefore $E \mid M_{0}$ has a local minimum at $\vec{\phi}_{0}$. Conversely, consider the curve $\omega \rightarrow \vec{\phi}_{\omega}$, and define

$$
\vec{\psi}(\omega ; x)=\vec{\phi}_{\omega}(x / \lambda(\omega)) \text {, where } \lambda^{n}(\omega)=Q\left(\vec{\phi}_{0}\right) / Q\left(\vec{\phi}_{\omega}\right) .
$$

Then $Q(\vec{\psi}(\omega))=Q\left(\vec{\phi}_{0}\right)$ and

$$
\begin{aligned}
E(\vec{\psi}(\omega)) & =L(\vec{\psi}(\omega))+\omega Q(\vec{\psi}(\omega)) \\
& =\omega Q\left(\vec{\phi}_{0}\right)+\int\left(\frac{1}{2} \lambda^{n-2}\left|\nabla \phi_{\omega}\right|^{2}+\lambda^{n}\left(F\left(\phi_{\omega}\right)-\frac{\omega^{2}}{2} \phi_{\omega}^{2}\right)\right) d x \\
& =\omega Q\left(\vec{\phi}_{0}\right)+\left(\frac{1}{2} \lambda^{n-2}-\left(\frac{1}{2}-\frac{1}{n}\right) \lambda^{n}\right) \int\left|\nabla \phi_{\omega}\right|^{2} d x .
\end{aligned}
$$

Thus, since $n \geqq 2$,

$$
E(\vec{\psi}(\omega)) \leqq \omega Q\left(\vec{\phi}_{0}\right)+d(\omega)=-\omega d^{\prime}\left(\omega_{0}\right)+d(\omega) .
$$

Now by assumption $E(\vec{\psi}(\omega)) \geqq E\left(\vec{\phi}_{0}\right)=d\left(\omega_{0}\right)-\omega_{0} d^{\prime}\left(\omega_{0}\right)$. So (18) implies

$$
d(\omega)-\omega d^{\prime}\left(\omega_{0}\right) \geqq d\left(\omega_{0}\right)-\omega_{0} d^{\prime}\left(\omega_{0}\right) .
$$

This means that $d(\omega)$ is convex at $\omega_{0}$.

Remark. If $\omega_{0}$ is a point of inflection of $d(\omega)$, then (by 10)) $\omega_{0}$ is a critical point of $Q\left(\vec{\phi}_{\omega}\right)$ and hence of $E\left(\vec{\phi}_{\omega}\right)$, since (by (9)) $\partial_{\omega} E\left(\vec{\phi}_{\omega}\right)=\omega \partial_{\omega} Q\left(\vec{\phi}_{\omega}\right)$.

\section{A Saddle Point of the Energy}

We view the solution $\vec{\phi}_{0}$ as a critical point of $E$ subject to constant $Q$. In (17) we defined a curve $\vec{\psi}(\omega)$ such that $\vec{\psi}\left(\omega_{0}\right)=\vec{\phi}_{0}$ and $Q(\vec{\psi}(\omega))=$ constant. We denote its first component by $\psi(\omega)=\phi_{\omega}(\cdot / \lambda(\omega))$ and its tangent vector at $\omega_{0}$ by

$$
y_{0}=\frac{\partial \psi}{\partial \omega}\left(\omega_{0}\right) \text { and } \vec{y}_{0}=\frac{\partial \vec{\psi}}{\partial \omega}\left(\omega_{0}\right)=\left[y_{0}, i\left(\omega_{0} y_{0}+\phi_{0}\right)\right]
$$

Note that $\omega \rightarrow \vec{\psi}(\omega)$ is a $C^{2}$ curve in $X$ because $\omega \rightarrow \vec{\phi}_{\omega}$ is $C^{2}$ and $\omega \rightarrow x \cdot \nabla \phi_{\omega}$ is 
continuous with values in $X$. An immediate consequence of the preceding theorem is

Corollary 6. (a) $\partial_{\omega}^{2} E\left(\vec{\psi}\left(\omega_{0}\right)\right) \leqq d^{\prime \prime}\left(\omega_{0}\right)$,

(b) if $d(\omega)$ is strictly concave at $\omega_{0}$, then $E(\vec{\psi}(\omega))<E\left(\vec{\phi}_{0}\right)$ for $\omega \neq \omega_{0}$, $\omega$ near $\omega_{0}$.

Proof. Part (b) is clear from the end of the proof of Theorem 5. Let $\alpha(\omega)=E(\vec{\psi}(\omega))-$ $d(\omega)+\omega d^{\prime}\left(\omega_{0}\right)$. Then $\alpha\left(\omega_{0}\right)=0$. By $(18), \alpha(\omega) \leqq 0$. Therefore $\alpha(\omega)$ has a maximum at $\omega_{0}$ and $\alpha^{\prime \prime}\left(\omega_{0}\right) \leqq 0$. This proves (a).

Remark 7. Consider the modified linearized operator for Eq. (2):

$$
T=-\Delta+f^{\prime}\left(\phi_{0}\right)-\omega_{0}^{2}+\frac{4 \omega_{0}^{2}}{\left(\phi_{0}, \phi_{0}\right)_{2}}\left(\phi_{0},\right)_{2} \phi_{0},
$$

where ()$_{2}$ denotes the ordinary $L^{2}$ inner product. If $d^{\prime \prime}\left(\omega_{0}\right)<0$, then $\left(T y_{0}, y_{0}\right)_{2}<0$. In order to prove this assertion, we use (11) to calculate

$$
\begin{aligned}
\partial_{\omega}^{2} E\left(\vec{\psi}\left(\omega_{0}\right)\right)= & \int\left\{\left|\omega_{0} y_{0}+\phi_{0}\right|^{2}+\operatorname{Re}\left(-\Delta+f^{\prime}\left(\phi_{0}\right)\right) y_{0} \cdot y_{0}\right. \\
& \left.+2 \omega_{0} \operatorname{Im} y_{0} \overline{i\left(\omega_{0} y_{0}+\phi_{0}\right)}\right\} d x \\
= & \int\left\{\left(-\Delta+f^{\prime}\left(\phi_{0}\right)\right) y_{0} \cdot y_{0}-\omega_{0}^{2} y_{0}^{2}+\phi_{0}^{2}\right\} d x \\
= & \left(T y_{0}, y_{0}\right)_{2} .
\end{aligned}
$$

By Corollary 6(a), this is negative if $d^{\prime \prime}\left(\omega_{0}\right)<0$. We will not need to use this remark, but it does put our analysis into the context of the linearized theory.

Lemma 8. (a) $\left\langle\left(E^{\prime \prime}\left(\vec{\phi}_{0}\right)-\omega_{0} Q^{\prime \prime}\left(\vec{\phi}_{0}\right)\right) \vec{y}_{0}, \vec{y}_{0}\right\rangle \leqq d^{\prime \prime}\left(\omega_{0}\right)$,

(b) $\left\langle Q^{\prime}\left(\vec{\phi}_{0}\right), \vec{y}_{0}\right\rangle=\int \phi_{0}^{2} d x+2 \omega_{0} \int \phi_{0} y_{0} d x=0$,

(c) $\int \nabla \phi_{0} \cdot \nabla y_{0}<0$ if $d^{\prime \prime}\left(\omega_{0}\right)<0$.

Proof. (a) follows immediately from Lemma 4 and Corollary 6. The charge

$$
Q(\vec{\psi}(\omega))=\omega \int|\psi(\omega)|^{2} d x
$$

is constant on the curve. Differentiating at $\omega=\omega_{0}$, we obtain (b). As for (c), we recall (17) to write

$$
\int|\nabla \psi(\omega)|^{2} d x=[\lambda(\omega)]^{n-2} \int\left|\nabla \phi_{\omega}\right|^{2} d x .
$$

Differentiating at $\omega_{0}$, we obtain

$$
2 \int \nabla \phi_{0} \cdot \nabla y_{0} d x=(n-2) \lambda^{n-3} \lambda^{\prime} \int\left|\nabla \phi_{0}\right|^{2} d x+n d^{\prime}\left(\omega_{0}\right) .
$$

But $d^{\prime}(\omega)<0$ and by (17) $\lambda^{n}=d^{\prime}\left(\omega_{0}\right) / d^{\prime}(\omega)$. Hence the signs of $\lambda^{\prime}\left(\omega_{0}\right)$ and of $d^{\prime \prime}\left(\omega_{0}\right)$ are the same.

Lemma 9. Let $L=\left\{\vec{u} \in X \mid\left\langle I(\vec{u}), i \vec{\phi}_{0}\right\rangle=0\right\}$ and $L_{\delta}=L \cap\left\{\vec{u} \in X \mid\left\|\vec{u}-\vec{\phi}_{0}\right\|<\delta\right\}$.If $\delta$ is small enough then

(i) $e^{i \theta} L_{\delta} \cap L_{\delta}=\phi$ for $0<\theta<2 \pi$, and

(ii) $U=\underset{\theta}{\cup} e^{i \theta} L_{\delta}$ is open in $X$. 
Proof. Let $F(\theta, \vec{v})=\left\langle I(\exp (-i \theta) \vec{v}), i \vec{\phi}_{0}\right\rangle$. Note that $F(\theta, \vec{v})=\sin \theta\left\langle I(\vec{v}), \vec{\phi}_{0}\right\rangle$ in case $\vec{v} \in L$. Choose $\delta$ small enough that $-\vec{v} \notin L_{\delta}$ whenever $\vec{v} \in L_{\delta}$. If $\vec{v} \in e^{i \theta} L_{\delta}$ with $0<\theta<2 \pi$, then $F(\theta, \vec{v})=0$. If also $\vec{v} \in L_{\delta}$ with $\delta$ small, then $\left\langle I(\vec{v}), \vec{\phi}_{0}\right\rangle \neq 0$. Hence $\sin \theta=0$, which is a contradiction. This proves (i). Now let $\vec{v} \in L_{\delta}$ and let $\vec{u} \in X$ belong to a neighborhood of $\vec{v}$. Let

$$
\alpha=\alpha(\vec{u})=\tan ^{-1} \frac{-\left\langle I(\vec{u}), i \vec{\phi}_{0}\right\rangle}{\left\langle I(\vec{u}), \vec{\phi}_{0}\right\rangle} .
$$

Then $\exp (-i \alpha) \vec{u} \in L$. By continuity of $\alpha$, it belongs to $L_{\delta}$ provided $\vec{u}$ is close enough to $\vec{v}$. This shows that $\vec{v}$ belongs to the interior of $U$. So $\exp (i \theta) \vec{v}$ belongs to the interior of $e^{i \theta} U=U$. Thus $U$ is an open set.

Proposition 10. There exists a $C^{1}$ functional $A: U \rightarrow \mathbb{R}$ such that

(i) $A\left(e^{i \theta} \vec{u}\right)=A(\vec{u})$ for $\vec{u} \in U$,

(ii) $A^{\prime}\left(\vec{\phi}_{0}\right)=-J \vec{y}_{0}$,

(iii) $A^{\prime}(\vec{u}) \in J[X] \subset X^{*}$ for $\vec{u} \in U$,

(iv) $\left\langle Q^{\prime}(\vec{u}), J^{-1} A^{\prime}(\vec{u})\right\rangle=0$ for $\vec{u} \in U$.

Proof. We first define $G: U \rightarrow L_{\delta}$ by

$$
G(\vec{u})=e^{-i \alpha(\vec{u})} \vec{u}
$$

where $\alpha(\vec{u})$ is given locally in the preceding proof. Clearly $G$ is smooth and $\alpha$ is locally smooth. We have $G(\exp (i \theta) \vec{u})=G(\vec{u})$. Next we define

$$
A(\vec{u})=-\left\langle J \vec{y}_{0}, G(\vec{u})\right\rangle .
$$

Property (i) is obvious. Now

$$
A^{\prime}(\vec{u})=e^{i \alpha(\vec{u})}\left\{-J \vec{y}_{0}+\left\langle J \vec{y}_{0}, i \vec{u}\right\rangle \alpha^{\prime}(\vec{u})\right\} .
$$

Since $\alpha\left(\vec{\phi}_{0}\right)=0$,

$$
\begin{aligned}
A^{\prime}\left(\vec{\phi}_{0}\right) & =-J \vec{y}_{0}+\left\langle J \vec{y}_{0}, i \alpha^{\prime}\left(\vec{\phi}_{0}\right) \vec{\phi}_{0}\right\rangle \\
& =-J \vec{y}_{0}-\left\langle Q^{\prime}\left(\vec{y}_{0}\right), \alpha^{\prime}\left(\vec{\phi}_{0}\right) \vec{\phi}_{0}\right\rangle \\
& =-J \vec{y}_{0}-\alpha^{\prime}\left(\vec{\phi}_{0}\right)\left\langle Q^{\prime}\left(\vec{\phi}_{0}\right), \vec{y}_{0}\right\rangle=-J \vec{y}_{0}
\end{aligned}
$$

by Lemma 8 (b). For (iii) we need by $(20)$ to show $\alpha^{\prime}(\vec{u})$ belongs to the range $R(J)$ of $J$. A direct calculation shows that $\alpha^{\prime}(\vec{u})$ is a linear combination of $\mathrm{I}\left(\vec{\phi}_{0}\right)$ and $\mathrm{I}\left(\vec{\phi}_{0}\right)$. Now $\mathrm{I}(\vec{v}) \in R(J)$ if and only if $v_{1} \in H_{r}^{1}$ and $v_{2} \in H_{r}^{1}$. Since $\phi_{0} \in H_{r}^{1}, \alpha^{\prime}(\vec{u}) \in R(J)$ and (iii) is proved. As for (iv), we have

$$
\begin{aligned}
0 & =\left.\frac{d}{d \theta} A\left(e^{i \theta} \vec{u}\right)\right|_{\theta=0}=\left\langle A^{\prime}(\vec{u}), i \vec{u}\right\rangle \\
& =\left\langle A^{\prime}(\vec{u}), J^{-1} Q^{\prime}(\vec{u})\right\rangle=-\left\langle Q^{\prime}(\vec{u}), J^{-1} A^{\prime}(\vec{u})\right\rangle .
\end{aligned}
$$

Lemma 11. Let $\gamma<\delta$ and let $\mathcal{O}=\bigcup_{\theta} e^{i \theta} L_{\gamma} \subset U$. Then there exists $\varepsilon>0$ and $a$ smooth function $S:(1-\varepsilon, 1+\varepsilon) \times \mathcal{O} \rightarrow U$ such that 
i) $S(1, \vec{u})=\vec{u}$,

ii) $\frac{\partial S}{\partial \lambda}(1, \vec{u})=-J^{-1} A^{\prime}(\vec{u})$,

iii) $Q(S(\lambda, \vec{u}))=Q(\vec{u})$.

If we denote $S(\lambda, \vec{u})=\vec{u}_{\lambda}=\left[u_{\lambda}, v_{\lambda}\right]$, we have

iv) $\int\left|\nabla u_{\lambda}\right|^{2} d x=\int\left|\nabla \phi_{0}\right|^{2} d x$ for some $\lambda=\lambda(\vec{u})$.

Proof. We solve the differential equation

$$
\frac{d \vec{u}_{\lambda}}{d \lambda}=-J^{-1} A^{\prime}\left(\vec{u}_{\lambda}\right), \quad \vec{u}_{1}=\vec{u} .
$$

Since $\vec{u} \rightarrow J^{-1} A^{\prime}(\vec{u})$ is well defined in $U$ and bounded on closed subsets of $U$ and since $\overline{\mathcal{O}} \subset U$, there is an $\varepsilon$-neighborhood in which (21) can be solved. We let $S$ be the flow $S(\lambda, \vec{u})=\vec{u}_{\lambda}$. By definition $S$ satisfies i) and ii).

We note that

$$
\partial_{\lambda} Q(S(\lambda, \vec{u}))=\left\langle Q^{\prime}\left(\vec{u}_{\lambda}\right),-J^{-1} A^{\prime}\left(\vec{u}_{\lambda}\right)\right\rangle=0
$$

by Proposition 10 (iv). Finally to show (iv) we calculate

$$
\frac{d \vec{u}_{\lambda}}{d \lambda}=-J^{-1} A^{\prime}\left(\vec{\phi}_{0}\right)=\vec{y}_{0} \quad \text { at } \lambda=1, \vec{u}=\vec{\phi}_{0}
$$

So by Lemma 8 (c) we have at $\lambda=1, \vec{u}=\vec{\phi}_{0}$,

$$
\frac{d}{d \lambda} \int\left|\nabla u_{\lambda}\right|^{2} d x=\operatorname{Re} \int \nabla \phi_{0} \nabla y_{0} d x \neq 0
$$

By the implicit function theorem we know that for $\vec{u}$ near $\vec{\phi}_{0}$, the equation

$$
\int\left|\nabla u_{\lambda}\right|^{2} d x=\int\left|\nabla \phi_{0}\right|^{2} d x
$$

has a solution $\lambda=\lambda(\vec{u})$. This completes the proof.

Proposition 12. Fix $\omega_{0}$ so that $d^{\prime \prime}\left(\omega_{0}\right)<0$. There is an $\varepsilon>0$ such that for all $\vec{u} \in \mathcal{O}, \vec{u} \neq e^{i \theta} \vec{\phi}_{0}$ for all $\theta, Q(\vec{u})=Q\left(\vec{\phi}_{0}\right)$, there is a $\lambda=\lambda(\vec{u}) \in(1-\varepsilon, 1+\varepsilon)$ such that

$$
E\left(\vec{\phi}_{0}\right)<E(\vec{u})+(\lambda-1) P(\vec{u}),
$$

where we denote

$$
P(\vec{u})=\left\langle E^{\prime}(\vec{u}),-J^{-1} A^{\prime}(\vec{u})\right\rangle .
$$

Proof. Let $\vec{u}_{\lambda}$ be the curve defined by Lemma 11. Then as in Lemma 4 we have

$$
\begin{aligned}
\left.\partial_{\lambda} E\left(\vec{u}_{\lambda}\right)\right|_{\lambda=1} & =\left\langle E^{\prime}(\vec{u}),-J^{-1} A^{\prime}(\vec{u})\right\rangle=P(\vec{u}), \\
\left.\partial_{\lambda}^{2} E\left(\vec{u}_{\lambda}\right)\right|_{\lambda=1} & =\left\langle E^{\prime \prime}(\vec{u}) \partial_{\lambda} \vec{u}, \partial_{\lambda} \vec{u}\right\rangle+\left\langle E^{\prime}(\vec{u}), \partial_{\lambda}^{2} \vec{u}\right\rangle, \\
0 & =\left\langle Q^{\prime \prime}(\vec{u}) \partial_{\lambda} \vec{u}, \partial_{\lambda} \vec{u}\right\rangle+\left\langle Q^{\prime}(\vec{u}), \partial_{\lambda}^{2} \vec{u}\right\rangle .
\end{aligned}
$$


These two equations imply for $\lambda=1, \vec{u}=\vec{\phi}_{0}$, that

$$
\partial_{\lambda}^{2} E\left(\vec{\phi}_{0}\right)=\left\langle\left(E^{\prime \prime}\left(\vec{\phi}_{0}\right)-\omega_{0} Q^{\prime \prime}\left(\vec{\phi}_{0}\right)\right) \vec{y}_{0}, \vec{y}_{0}\right\rangle \text {. }
$$

By Lemma 8(a) we have

$$
\partial_{\lambda}^{2} E\left(\vec{\phi}_{0}\right) \leqq d^{\prime \prime}\left(\omega_{0}\right)<0,
$$

so that the Taylor expansion gives

$$
E\left(\vec{u}_{\lambda}\right)<E(\vec{u})+(\lambda-1) P(\vec{u})
$$

near $\vec{u}=\vec{\phi}_{0}, \lambda=1$. On the other hand, as in (15) we have

$$
E\left(\vec{u}_{\lambda}\right) \geqq \omega_{0} Q\left(\vec{u}_{\lambda}\right)+L\left(u_{\lambda}, i \omega_{0} u_{\lambda}\right) .
$$

We have $Q\left(\vec{u}_{\lambda}\right)=Q\left(\vec{\phi}_{0}\right)$. We choose $\lambda=\lambda(\vec{u})$ as in Lemma 11 (iv). Thus $\int\left|\nabla u_{\lambda}\right|^{2} d x=$ $\int\left|\nabla \phi_{0}\right|^{2} d x$. By Theorem 2(e), $L\left(u_{\lambda}, i \omega_{0} u_{\lambda}\right) \geqq L\left(\vec{\phi}_{0}\right)$.

Hence

$$
E\left(\vec{u}_{\lambda}\right) \geqq \omega_{0} Q\left(\vec{\phi}_{0}\right)+L\left(\vec{\phi}_{0}\right)=E\left(\vec{\phi}_{0}\right) .
$$

A comparison with (25) completes the proof in case $\vec{u}$ is near $\vec{\phi}_{0}$. If $\vec{u}$ is near $\exp (i \theta) \vec{\phi}_{0}$, then we apply this result to $\exp (-i \theta) \vec{u}$.

Corollary 13. The curve $\vec{\psi}(\omega)$ satisfies:

(i) $E(\vec{\psi}(\omega))<E\left(\vec{\phi}_{0}\right)$ for $\omega \neq \omega_{0}$,

(ii) $Q(\vec{\psi}(\omega))=Q\left(\vec{\phi}_{0}\right)$, and

(iii) $P\left(\vec{\psi}\left(\omega_{0}\right)\right)$ changes sign as $\omega$ passes $\omega_{0}$.

Proof. We noted (i) in Corollary 6, while (ii) is obvious from (17). By Lemma 8(c)).

$$
\frac{d}{d \omega} \int|\nabla \psi(\omega)|^{2} d x \neq 0 \text { at } \omega=\omega_{0}
$$

Therefore $\int|\nabla \psi(\omega)|^{2} d x-\int\left|\nabla \phi_{0}\right|^{2} d x$ changes sign at $\omega_{0}$, so that $\lambda-1=$ $\lambda(\vec{\psi}(\omega))-1$ also changes sign. Applying Proposition 12 to $\vec{u}=\vec{\psi}(\omega),(\lambda-1) P(\vec{\psi}(\omega))$ $>0$, so that $P(\vec{\psi}(\omega))$ also changes sign.

\section{The Evolution Equation}

Now consider NLKG, Eq. (1) or equivalently

$$
J \frac{d \vec{u}}{d t}=E^{\prime}(\vec{u}), \quad \vec{u}(0)=\vec{u}_{0} .
$$

Formally, the energy and charge are conserved quantities:

$$
E(\vec{u}(t))=E\left(\vec{u}_{0}\right), \quad Q(\vec{u}(t))=Q\left(\vec{u}_{0}\right) .
$$

In fact, for any $\vec{u}_{0} \in X$, Eq. (7) has a unique strong solution [18] $u \in C([0, T) ; X)$ satisfying (26) for some $T>0$, under the growth condition $(H 2)$. We fix a frequency $\omega_{0}$ with $d^{\prime \prime}\left(\omega_{0}\right)<0$ and denote $E_{0}=E\left(\vec{\phi}_{0}\right), Q_{0}=Q\left(\vec{\phi}_{0}\right)$. Let $K=\left\{\exp (i \theta) \vec{\phi}_{0} \mid \theta \in \mathbb{R}\right\}$ 
and let $N=\mathcal{O} \backslash K$, where $\mathcal{O}$ is the tubular neighborhood of $K$ defined in Lemma 11. We continue to assume $f$ satisfies $(H 1)$ and $(H 2)$, but for simplicity we also assume $f \in C^{\infty}$.

Lemma 14. The sets

$$
\begin{aligned}
& S_{1}=\left\{\vec{u} \in N \mid E(\vec{u})<E_{0}, Q(\vec{u})=Q_{0}, P(\vec{u})>0\right\} \\
& S_{2}=\left\{\vec{u} \in N \mid E(\vec{u})<E_{0}, Q(\vec{u})=Q_{0}, P(\vec{u})<0\right\}
\end{aligned}
$$

are invariant regions under the flow of (7). In particular, if $\vec{u}_{0} \in S_{1}$, then $P(\vec{u}(t))>0$ for all $t>0$ such that $\vec{u}(s) \in N$ for $0 \leqq s \leqq t$; if $\vec{u}_{0} \in S_{2}$, the inequality is reversed.

Proof. Since $E$ and $Q$ are conserved, we have by Proposition 12, if $\vec{u}(s) \in N$ for $0 \leqq s \leqq t$

$$
0 \leqq E_{0}-E(\vec{u}(t))<(\lambda-1) P(\vec{u}(t)),
$$

where $\lambda$ depends on $t$. Therefore $P(\vec{u}(t)) \neq 0$. By continuity of the solution curve $\left(P(\vec{u}(t))\right.$ has one sign. Consequently $S_{1}$ and $S_{2}$ are invariant regions. Note that $S_{1}$ and $S_{2}$ each contain points arbitrarily close to $\vec{\phi}_{0}$, by Corollary 13 .

Lemma 15. Let $\vec{u}_{0} \in S_{1}$ (respectively $S_{2}$ ) and let

$$
T_{0}=\sup \{t \mid \vec{u}(s) \in N, 0 \leqq s<t\} \leqq \infty
$$

be the exit time. Then there is positive $\varepsilon_{0}$ such that $P(\vec{u}(t))>\varepsilon_{0}$ (respectively $<-\varepsilon_{0}$ for $t<T_{0}$ ).

Proof. From Proposition 12 we have

$$
E_{0}-E(\vec{u}(t)) \leqq(\lambda-1) P(\vec{u}(t)) \text { for } 0 \leqq t<T_{0} .
$$

Let $\varepsilon_{0}=E_{0}-E\left(u_{0}\right)$. Since $u_{0} \in S_{1} \cup S_{2}$, we have $\varepsilon_{0}>0$. So by (24), $\varepsilon_{0} \leqq$ $(\lambda-1) P(\vec{u}(t))$. Since $\vec{u}(t) \in N$, we have $|\lambda-1|<\delta<1$, whence $0<\lambda<2$. If $\vec{u}_{0} \in S_{1}$, then by Lemma $14, P(\vec{u}(t))>0$. Hence $\lambda>1$ and $P(\vec{u}(t))>\varepsilon_{0} /(\lambda-1)>\varepsilon_{0}$. A similar argument shows that $P(\vec{u}(t))<-\varepsilon_{0}$ if $\vec{u}_{0} \in S_{2}$.

Theorem 16. If $d^{\prime \prime}\left(\omega_{0}\right)<0$ then $K=\left\{e^{i \theta} \vec{\phi}_{0}\right\}$ is unstable under the flow of (7). Specifically, if $\vec{u}_{0} \in S_{1} \cup S_{2}$ (consequently $\vec{u}_{0}$ is arbitrarily close to $K$ ), the solution with initial condition $\vec{u}(0)=\vec{u}_{0}$ exits $N$ in finite time: $T_{0}<\infty$.

Proof. A bootstrap argument shows that $\phi_{\omega} \in C^{\infty}$, while the fact that $1-\omega^{2}>0$ shows that $\phi_{\omega}(x)$ decays exponentially as $|x| \rightarrow \infty$. (See [13] or [3].) Since $\partial \phi_{\omega} / \partial \omega$ satisfies the elliptic equation

$$
\left[-\Delta+f^{\prime}\left(\phi_{\omega}\right)-\omega^{2}\right] \frac{\partial \phi_{\omega}}{\partial \omega}=2 \omega \phi_{\omega}
$$

it too is $C^{\infty}$ and exponentially decaying. Therefore so is $\vec{y}_{0}=\partial \vec{\psi}(\omega) / \partial \omega \mid \omega=\omega_{0}$. By Eq. (20), the range of $A^{\prime}(\vec{u})$ can be identified with smooth, exponentially decaying functions, which of course belong to every Sobolev space. This justifies the following procedure. 
Let $\vec{u}_{0} \in S_{1} \cup S_{2}$. Apply Eq. (7) to $-J^{-1} A^{\prime}(\vec{u}(t)) \in X$ to obtain

$$
\left\langle J \frac{d \vec{u}}{d t},-J^{-1} A^{\prime}(\vec{u}(t))\right\rangle=\left\langle E^{\prime}(\vec{u}(t)),-J^{-1} A^{\prime}(\vec{u}(t))\right\rangle .
$$

By (23) and since $J^{*}=-J$, this can be written as

$$
\frac{d}{d t} A(\vec{u}(t))=\left\langle A^{\prime}(\vec{u}(t)), \frac{d u}{d t}\right\rangle=P(\vec{u}(t)) .
$$

By Lemma $15, \pm P(\vec{u}(t))>\varepsilon_{0}$ so long as $\vec{u}(t) \in N$. So by (27),

$$
\left|A(\vec{u}(t))-A\left(\vec{u}_{0}\right)\right|>\varepsilon_{0} t .
$$

Since $N$ is a bounded set and $A$ is bounded on $N$, the solution must exit from $N$ in a finite time.

\section{Examples}

We begin with the pure power case

$$
f(u)=u-|u|^{p-1} u, \quad 1<p<1+4 /(n-2) .
$$

The homogeneity of this nonlinear term permits the adjustment of coefficients by scaling. The stationary Eq. (2) is

$$
-\Delta \phi+\left(1-\omega^{2}\right) \phi-|\phi|^{p-1} \phi=0, \quad-1<\omega<1 .
$$

By dilation and scaling, every solution $\phi=\phi_{\omega}$ has the form

$$
\phi_{\omega}(x)=\lambda^{2 /(p-1)} v(\lambda x)
$$

where $\lambda=\left(1-\omega^{2}\right)^{1 / 2}$ and

$$
-\Delta v+v-|v|^{p-1} v=0, \quad v=\phi_{0} .
$$

With this explicit dependence of $\phi_{\omega}$ on $\omega$, we can easily calculate all the relevant quantities. Thus,

$$
d(\omega)=\frac{1}{n} \int\left|\nabla \phi_{\omega}\right|^{2} d x=\frac{1}{n} \lambda^{a} \int|\nabla v|^{2} d x=\lambda^{a} d(0),
$$

where $a=4 /(p-1)-n+2$. Note that $a>0$ because of the restriction on $p$. Differentiating twice, we find

$$
d^{\prime \prime}(\omega)=a\left((a-1) \omega^{2}-1\right)\left(1-\omega^{2}\right)^{(a-4) / 2} d(0) .
$$

Case 1. If $p \geqq 1+4 / n$, then $a-1 \geqq 1, d(\omega)$ is concave for all $\omega^{2}<1$, and $\phi_{\omega}$ is always unstable.

Case 2. If $p<1+4 / n$, then $a-1<1$ and $d^{\prime \prime}(\omega)$ changes sign at $\omega_{c}=(a-1)^{-1 / 2}$. Therefore $\phi_{\omega}$ is stable for $\omega_{c}<|\omega|<1$ and unstable for $|\omega|<\omega_{c}$.

Using the fact [13] that

$$
\frac{1}{n} \int|\nabla v|^{2} d x=\frac{1}{a} \int v^{2} d x=\frac{1}{n+a} \int v^{p+1} d x
$$


we easily calculate

$$
\begin{aligned}
d(\omega) & =\left(1-\omega^{2}\right)^{a / 2} a^{-1} \int v^{2} d x, \\
Q\left(\vec{\phi}_{\omega}\right) & =\omega\left(1-\omega^{2}\right)^{(a-2) / 2} \int v^{2} d x, \\
E\left(\vec{\phi}_{\omega}\right) & =\left[\omega^{2}\left(1-\omega^{2}\right)^{(a-2) / 2}+a^{-1}\left(1-\omega^{2}\right)^{a / 2}\right] \int v^{2} d x .
\end{aligned}
$$

Thus, in Case 2, as $\omega$ goes from 0 to $1, Q$ rises from zero to a maximum at $\omega_{c}$ and falls back to zero, while $E$ rises from $E\left(\vec{\phi}_{0}\right)$ to a maximum at $\omega_{c}$ and falls back to zero. If $E$ is plotted against $Q$, the unstable part lies above the stable part and they are joined by a cusp at the critical frequency $\omega_{c}$.

In the pure power case we can make a more explicit construction which gives an alternative proof of the main theorem. (This was our original proof.) In fact, the curve $\omega \rightarrow \phi_{\omega}$ is given by (28). Hence $\psi$, defined by (17), has the form $\psi(\omega ; x)=\alpha(\omega) \phi_{\omega_{0}}(\beta(\omega) x)$. Therefore

$$
y_{0}(x)=\beta^{\prime}\left(\omega_{0}\right) x \cdot \nabla \phi_{\omega_{0}}(x)+\alpha^{\prime}\left(\omega_{0}\right) \phi_{\omega_{0}}(x) .
$$

This indicates that, instead of constructing the operator $A$, we could directly use the multiplier

$$
M u=x \cdot \nabla u+\frac{2}{p-1} u
$$

to take the place of $-J^{-1} A^{\prime}(\vec{u})$. Then we would let $P(\vec{u})=-n K(\vec{u})+2(p-1)^{-1}$ $R(\vec{u})$, where $K$ is the functional associated with dilations and is given by Definition 1 , and $R$ is associated with scaling and is given by

$$
R(\vec{u})=\int\left(-\left|u_{2}\right|^{2}+\left|\nabla u_{1}\right|^{2}+\bar{u}_{1} f\left(u_{1}\right)\right) d x .
$$

Lemmas 9, 10, 11 can be omitted and Proposition 12 suitably modified. Then Corollary 13 and Lemmas 14 and 15 are valid as stated. Finally in the proof of Theorem 16, Eq. (27) is replaced by the "broken dilation identity," which comes from multiplying Eq. (1) by $\mathrm{Mu}$ :

$$
\frac{d^{2}}{d t^{2}} \int\left\{\left(r^{2} \frac{1}{2}\left|u_{t}\right|^{2}+\frac{1}{2}|\nabla u|^{2}+F(u)\right)-\frac{2}{p-1}|u|^{2}\right\} d x=2 P(u) .
$$

Note that the integral on the left brings us outside the Hilbert space $X$. On the other hand it is "almost" non-negative. Therefore it is possible to complete the proof that the solution exits $N$ in a finite time. We believe that this identity (32) will find other uses.

As a second example, consider the mixed power

$$
f(u)=u-\alpha|u|^{q-1} u+|u|^{p-1} u,
$$

where $\alpha>0$ and $1<p<1+4 /(n-2)$. For large enough $\alpha$, there is a non-empty interval $\omega^{*}<|\omega|<1$ which satisfies $(H 1)$. It can be shown [10] that $d(\omega) \rightarrow \infty$ as $\omega \downarrow \omega^{*}$, that $d(\omega)$ is convex near $\omega^{*}$, that $d(\omega)$ is decreasing and that it is concave near $\omega=1$. Therefore there exist both stable and unstable frequencies.

Anderson [1] studied the particular case

$$
f(u)=u-|u|^{2} u+|u|^{4} u, \quad x \in \mathbb{R}^{3}
$$


numerically. Even though $(H 2)$ is not satisfied, and uniqueness of solutions of NLKG is not known, weak solutions do exist for all time, due to the positivity of the term $|u|^{4} u$. (See [12] and Sect. 6 below.) In this case $\omega^{*}=13 / 16$. For the stable frequencies, see [10].

As a third example, consider the case of zero frequency $\omega=0$. Let $f$ be any function which satisfies $(H 1)$ and $(H 2)$ with $\omega=0$. This case was treated in [11], where a different method was used to prove the instability. Although the method of [11] has certain advantages, we wish to show how the $\omega=0$ case fits into the context of this paper. Indeed, $\vec{\phi}_{0}=\left[\phi_{0}, 0\right]$ and $d^{\prime \prime}(0)=-\int \phi_{0}^{2} d x<0$ by Lemma 3. The curve $\vec{\psi}$ is defined by simple dilation as $\vec{\psi}(\alpha ; x)=\left[\phi_{0}(\vec{x} / \alpha), 0\right]$. Then $Q(\vec{\psi}(\alpha))=0$ and

$$
E(\vec{\psi}(\alpha))=\alpha^{n-2} \int \frac{1}{2}\left|\nabla \phi_{0}\right|^{2} d x+\alpha^{n} \int F\left(\phi_{0}\right) d x .
$$

Hence $\quad \dot{E}(1)=0$ and $\ddot{E}(1)=-(n-2) \int\left|\nabla \phi_{0}\right|^{2} d x<0$. Furthermore, $\vec{y}_{0}=$ $\left[-x \cdot \nabla \phi_{0}, 0\right]$. The rest of the proof is unchanged.

\section{The Schrödinger Equation}

In this section we prove that with only minor modifications the instability theorem is also valid for the equation

$$
i \frac{\partial u}{\partial t}-\Delta u+f(u)=0
$$

where $f(0)=f^{\prime}(0)=0, f\left(e^{i \theta} u\right)=e^{i \theta} f(u)$. This equation has $u(x, t)=\phi(x) \exp (i \omega t)$ as a solution if

$$
-\Delta \phi+f(\phi)-\omega \phi=0
$$

We assume that $f$ satisfies $(H 2)$ and

$$
F(\eta)-\frac{1}{2} \omega \eta^{2}<0 \text { for some } \eta \text { and some } \omega<0 \text {. }
$$

Thus $\omega$ satisfies $-\infty<\omega<\omega^{*} \leqq 0$. With some changes of notation, Theorem 2 asserts the existence of a solution $\phi_{\omega}(x)$ of (34). We make the following definitions. Let $X=H_{r}^{1}$, considered as a real Hilbert space. Let $I$ and $J$ be the mappings: $X \rightarrow X^{*}$ given by

$$
\langle I(u), v\rangle=\operatorname{Re} \int u \bar{v} d x,\langle J u, v\rangle=\operatorname{Im} \int u \bar{v} d x .
$$

Thus $J u=I(-i u)$. Let

$$
\begin{aligned}
E(u) & =\int\left(\frac{1}{2}|\nabla u|^{2}+F(u)\right) d x, \\
Q(u) & =\frac{1}{2} \int|u|^{2} d x, \\
L(u, v) & =\frac{1}{2} \operatorname{Im} \int u \bar{v} d x+E(u) .
\end{aligned}
$$

Then Eq. (33) takes the form $J(d u / d t)=E^{\prime}(u)$ and (34) takes the form

$$
E^{\prime}(\phi)=i \omega J \phi=\omega Q^{\prime}(\phi)
$$


as in Sect. 1. We have

$$
d(\omega)=L\left(\phi_{\omega}, i \omega \phi_{\omega}\right)=\frac{1}{n} \int\left|\nabla \phi_{\omega}\right|^{2} d x
$$

and $d^{\prime}(\omega)=-Q\left(\phi_{\omega}\right)$ as in Lemma 3 . The proofs from Lemma 3 to Theorem 16 are valid with only cosmetic changes. Indeed, inequality (15) is replaced by the equality

$$
E(u)=L(u, i \omega u)+\omega Q(u) .
$$

As before, we work with the curve $\psi(\omega)=\phi_{\omega}(\cdot / \lambda(\omega))$, where $\lambda(\omega)$ is chosen so that $Q(\psi(\omega))=Q\left(\phi_{\omega_{0}}\right)$. We define $y_{0}=\partial \psi / \partial \omega$ at $\omega=\omega_{0}$. We finally obtain the following analogue of Theorem 16.

Theorem 17. If $d^{\prime \prime}\left(\omega_{0}\right)<0$, then $K=\left\{e^{i \theta} \phi_{\omega_{0}}\right\}$ is unstable under the flow of (33). Specifically there is a neighborhood $N$ of $K$ such that, if $u_{0} \in X, E\left(u_{0}\right)<E\left(\phi_{\omega_{0}}\right)$, $Q\left(u_{0}\right)=Q\left(\phi_{\omega_{0}}\right)$ and $P\left(u_{0}\right) \neq 0$ (so that $u_{0}$ may be arbitrarily near $K$ ), the solution of (33) with initial condition $u_{0}$ exits $N$ in a finite time.

The corresponding stability theorem, which follows from Theorem 6, may be found in [4].

For example, take the pure power case

$$
f(u)=-|u|^{p-1} u \text { with } 1<p<1+4 /(n-2) .
$$

Then $\phi_{\omega}$ is given by (28) where $\lambda=\sqrt{-\omega}$. Also $d(\omega)=\lambda^{a} d(0)$ and $d^{\prime \prime}(\omega)=$ $a(a-2) \lambda^{a-4} d(0) / 4$. Hence we have instability for all $\omega<0$ if $p>1+4 / n$ and stability for all $\omega<0$ if $p<1+4 / n$. An example where the stability depends on $\omega$, as in Sect. 4 , can also be given.

\section{Extensions}

This section has two parts. First we show that the ground state $\phi_{\omega}$ depends smoothly on $\omega$ if it is unique. Secondly we discuss rapidly growing nonlinearities.

I. If the ground state $\phi_{\omega}$ (defined in Theorem 2(a)) is not unique, then the set of ground states is still stable or unstable according to the convexity or concavity of $d(\omega)$, but it is rather cumbersome to formulate so we shall exclude that case from consideration.

Theorem 18. For $\omega$ near $\omega_{0}$, let $\phi_{\omega}$ be the unique real solution of the minimum problem stated in Theorem 2(d). Let zero not be eigenvalue of the linearized operator $\mathscr{L}_{0}=-\Delta-\omega_{0}^{2}+f_{0}^{\prime}\left(\phi_{\omega_{0}}\right)$ at $\phi_{\omega_{0}}$ acting on $L_{r}^{2}$ (real-valued, radial functions in $L^{2}\left(\mathbb{R}^{n}\right)$ ). Iff is a $C^{2}$ function satisfying $(H 1)$ and $(H 2)$, then $\omega-\vec{\phi}_{\omega}$ is a $C^{2}$ mapping of $\mathbb{R}$ into $X$ for $\omega$ near $\omega_{0}$.

Lemma 19. $\omega \rightarrow \vec{\phi}_{\omega}$ is continuous with values in $X$.

Proof. From Proposition 2.1 of $[10], d(\omega)=n^{-1} \int\left|\nabla \phi_{\omega}\right|^{2} d x$ is a continuous function of $\omega$. From Lemma 2.1 of [10], $\int \phi_{\omega}^{2} d x$ is a bounded function of $\omega$. Let $\left(\omega_{j}\right)$ be a sequence tending to $\omega_{0}$. Then $\left(\phi_{\omega_{i}}\right)$ is bounded in $H_{r}^{1}$. A subsequence 
may be chosen converging weakly in $H_{r}^{1}$ to some $v$. Now

$$
0=K\left(\phi_{\omega}, i \omega \phi_{\omega}\right)=\int\left[\left(\frac{1}{2}-\frac{1}{n}\right)\left|\nabla \phi_{\omega}\right|^{2}+\frac{1-\omega^{2}}{2} \phi_{\omega}^{2}+G\left(\phi_{\omega}\right)\right] d x,
$$

where $G(s)=F(s)-s^{2} / 2$. Letting $\omega=\omega_{j} \rightarrow \omega_{0}$, the weak limit and (H2) imply that $K(v, i \omega v) \leqq 0$. This is the constraint in the definition of $d(\omega)$ in Theorem 2 . Therefore $\int|\nabla v|^{2} d x \geqq \int\left|\nabla \phi_{\omega_{0}}\right|^{2} d x$. By weak limits, this is an equality. By the uniqueness assumption in Theorem 18,v $=\phi_{\omega_{0}}$. It follows that $\phi_{\omega}$ tends weakly to $\phi_{\omega_{0}}$ in $H_{r}^{1}$. The continuity of $d(\omega)$ implies that $\int\left|\nabla\left(\phi_{\omega}-\phi_{\omega_{0}}\right)\right|^{2} d x \rightarrow 0$. Passage to the limit in the constraint $K_{\omega}\left(\phi_{\omega}\right)=0$ yields $\phi_{\omega} \rightarrow \phi_{\omega_{0}}$ in $L^{2}$. Hence $\vec{\phi}_{\omega} \rightarrow \vec{\phi}_{\omega_{0}}$ in $X$.

Lemma 20. In a neighborhood in $X$ of $\vec{\phi}_{\omega_{0}}$, all the solutions lie on a $C^{1}$ curve.

Proof. We write Eq. (2) as

$$
-\Delta \phi+\lambda \phi+g(\phi)=0
$$

where $\lambda=1-\omega^{2}>0$ and $g(s)=f(s)-s$. Let $\lambda_{0}=1-\omega_{0}^{2}$ and $\phi_{0}=\phi_{\omega_{0}}$. Let

$$
\mathscr{G}(\lambda, u)=u-(\lambda-\Delta)^{-1} g(u), \quad \lambda>0, \quad u \in H_{r}^{1} .
$$

Then $\mathscr{G}(\lambda, u) \in H_{r}^{1}$ since $u \in H^{1} \subset L^{2 n /(n-2)}, g(u) \in L^{2 n /(n+2)}$ by $(H 2)$ and $(\lambda-\Delta)^{-1}$ $g(u) \in H^{1}$ by Sobolev's embedding. In fact $\mathscr{G}$ is a $C^{1}$ operator from $(0, \infty) \times H_{r}^{1}$ into $H_{r}^{1}$ Note that $\mathscr{G}\left(\lambda_{0}, \phi_{0}\right)=0$. Now the operator $\mathscr{L}_{0}=-\Delta+\lambda_{0}+g^{\prime}\left(\phi_{0}\right)$ acting on $L_{r}^{2}$, has only discrete spectrum to the left of $\lambda_{0}$, and we have assumed it is invertible. It follows that the compact operator $\left(\lambda_{0}-\Delta\right)^{-1 / 2} g^{\prime}\left(\phi_{0}\right)\left(\lambda_{0}-\Delta\right)^{-1 / 2}$ on $L_{r}^{2}$ does not have -1 in its spectrum. Hence

$$
\frac{\partial \mathscr{G}}{\partial u}\left(\lambda_{0}, \phi_{0}\right)=I+\left(\lambda_{0}-\Delta\right)^{-1} g^{\prime}\left(\phi_{0}\right)
$$

acting from $H_{r}^{1}$ to $H_{r}^{1}$, is also invertible. By the implicit function theorem, the solutions of $\mathscr{G}(\lambda, u)=0$ in a neighborhood of $\left(\lambda_{0}, \phi_{0}\right)$ form a $C^{1}$ curve in $(0, \infty) \times H_{r}^{1}$.

Proof of Theorem 18. By Lemmas 19 and $20, \omega \rightarrow \phi_{\omega}$ is a $C^{1}$ curve near $\omega=\omega_{0}, \phi_{\omega}=\phi_{0} \in H_{r}^{1}$. Since $\phi_{\omega}$ is bounded in $H_{r}^{1}$, the functions $|x|^{(n-1) / 2} \phi_{\omega}(x)$ are bounded independently of $\omega$ and $x$ (see [13]). By a bootstrap argument using the elliptic Eq. (2), $\phi_{\omega}(x)$ is uniformly bounded. Now $\phi_{\omega}^{\prime}=\partial \phi_{\omega} / \partial \omega$ satisfies the elliptic equation

$$
\left[-\Delta-\omega^{2}+f^{\prime}\left(\phi_{\omega}\right)\right] \phi_{\omega}^{\prime}=2 \omega \phi_{\omega} .
$$

Since $\mathscr{L}_{0}$ is invertible, $\phi_{\omega}^{\prime}(x)$ is also uniformly bounded. Therefore $\left[\phi_{\omega}^{\prime}(x)\right]^{2}$ is bounded in $H^{1}$. Now we can show that $\phi_{\omega}$ is $C^{2}$ with values in $H^{1}$ by taking difference quotients in the last equation. It follows that $\phi_{\omega}^{\prime \prime}=\partial \phi_{\omega}^{\prime} / \partial \omega$ exists and satisfies

$$
\left[-\Delta-\omega^{2}+f^{\prime}\left(\phi_{\omega}\right)\right] \phi_{\omega}^{\prime \prime}=4 \omega \phi_{\omega}^{\prime}+2 \phi_{\omega}-f^{\prime \prime}\left(\phi_{\omega}\right)\left[\phi_{\omega}^{\prime}\right]^{2} .
$$

This completes the proof. 
II. Now we consider a nonlinearity $f$ which grows more rapidly than allowed by $(H 2)$. In fact, we replace $(H 2)$ by

$(H 2)_{p} \quad$ There exists $\delta>0$ and $p \geqq 1+4 /(n-2)$ such that

$$
f(s) \geqq \delta s^{p} \text { and }\left|f^{\prime}(s)\right| \leqq \delta^{-1}|s|^{p-1}
$$

for sufficiently large $s$.

We define $X=\left(H_{r}^{1} \cap L^{p+1}\right) \oplus L_{r}^{2}$, considered as a real Banach space. Then $E$ and $Q$ are $C^{2}$ functionals on $X$. Sections 1 and 2 of this paper are completely unchanged. The analogue of Theorem 16 is

Theorem 21. If $d^{\prime \prime}\left(\omega_{0}\right)<0$ and $\vec{u}_{0} \in S_{1} \cup S_{2}$, then there exists a solution $\vec{u}(t)$ of Eq (7), which is weakly continuous in $t$ with values in $X$ and which exits $N$ in a finite time. (This is valid for NLS as well.)

Proof. It is not known whether there are strong solutions of the evolution equation for arbitrary data in $X$. However it is known [12] that, for each $\vec{u}_{0} \in X$, there is at least one weak solution $\vec{u} \in C_{w}([0, \infty) ; X)$, with $E(\vec{u}(t)) \leqq E\left(\vec{u}_{0}\right)$ for all $t>0 .\left(C_{w}\right.$ denotes the space of weakly continuous functions.)

The weak solution satisfies $Q(\vec{u}(t))=Q\left(\vec{u}_{0}\right)$ for all $t>0$. This is proved as follows. The solution $\vec{u}$ is the weak limit of solutions $u^{\varepsilon}$ of a certain regularized equation (see [12]). Multiplying it by $\bar{u}^{\varepsilon} \zeta$, where $\zeta=\zeta(x)$ is a test function, and taking the imaginary part, we have

$$
\left.\operatorname{Im} \int \partial_{t} u^{\varepsilon} \bar{u}_{\varepsilon} \zeta d x\right|_{0} ^{t}+\int_{0}^{t} \nabla u^{\varepsilon} \cdot \nabla \zeta \bar{u}^{\varepsilon} d x=0
$$

Now let $\partial_{t} u^{\varepsilon} \rightarrow \partial_{t} u$ and $\nabla u^{\varepsilon} \rightarrow \nabla u$ weakly in $L^{2}$ but $u^{\varepsilon} \rightarrow u$ strongly in $L_{\text {loc }}^{2}$. Then the same identity is valid for the limit $u$. Finally we remove the test function $\zeta(x) \rightarrow 1$ to obtain

$$
\left.\operatorname{Im} \int \partial_{t} u \bar{u} d x\right|_{0} ^{t}=0
$$

Next we note that $P(\vec{u}(t))$ is a continuous function of $t$. Indeed, recall that

$$
P(\vec{u}(t))=\left\langle E^{\prime}(\vec{u}(t)),-J^{-1} A^{\prime}(\vec{u}(t))\right\rangle .
$$

From Eq. (20) it is clear that $A^{\prime}: X \rightarrow X^{*}$ is completely continuous. Hence $t \rightarrow A^{\prime}(\vec{u}(t))$ is strongly continuous with values in $X^{*}$, and $t \rightarrow J^{-1} A(\vec{u}(t))$ with values in $X$. The other factor in $(23), E^{\prime}(\vec{u}(t))$, is weakly continuous with values in $X^{*}$. Therefore $P(\vec{u}(t))$ is continuous.

The rest of Sect. 3 continues almost verbatim. We should just be careful to write $(27)$ in its integrated form

$$
A(\vec{u}(t))-A\left(\vec{u}_{0}\right)=\int_{0}^{t} P(\vec{u}(s)) d s .
$$

Clearly $(H 2)_{p}$ is not the sharpest possible hypothesis. 


\section{References}

1. Anderson, D.: Stability of time-dependent particlelike solutions in nonlinear field theories II. J. Math. Phys. 12, 945-952 (1971)

2. Berestycki, H., Cazenave, T.: Instabilité des états stationnaires dans les équations de Schrödinger et de Klein-Gordon non linéaires. C.R. Acad. Sci. 293, 489-492 (1981)

3. Berestycki, H., Lions, P. L.: Nonlinear scalar field equations. Arch. Rat. Mech. Anal. 82, 313-375 (1983)

4. Cazenave, T., Lions, P. L.: Orbital stability of standing waves for some nonlinear Schrödinger equations. Commun. Math. Phys. 85, 549-561 (1982)

5. Glassey, R.: On the blowing-up of solutions to the Cauchy problem for nonlinear Schrödinger equations. J. Math. Phys. 18, 1794-7 (1977)

6. Keller, C.: Stable and unstable manifolds for the nonlinear wave equation with dissipation. J. Diff. Eqs. 50, 330-347 (1983)

7. Lee, T. D.: Particles physics and introduction to field theory. New York: Harwood Academic 1981

8. Makhankov, V. G.: Dynamics of classical solutions (in non-integrable systems). Phys. Rep. 35, 1-128 (1978)

9. Payne, L., Sattinger, D.: Saddle points and instability of nonlinear hyperbolic equations. Israel J. Math. 22, 273-303 (1975)

10. Shatah, J.: Stable standing waves of nonlinear Klein-Gordon equations. Commun. Math. Phys. 91, 313-327 (1983)

11. Shatah, J.: Unstable ground states of nonlinear Klein-Gordon equations. Trans. A.M.S. (1985)

12. Strauss, W.: On weak solutions of semi-linear hyperbolic equations. Anais Acad. Brasil. Cienc. 42, 645-651 (1970)

13. Strauss, W.: Existence of solitary waves in higher dimensions. Commun. Math. Phys. 55, 149-162 (1977)

14. Strauss, W.: Stable and unstable states of nonlinear wave equations. Contemp. Math. 17, 429-441 (1983)

15. Weinstein, M.: Stability analysis of ground states of nonlinear Schrödinger equations. preprint

16. Berestycki, H., Gallouet, T., Kavian, O.: Equations des champs scalaires euclidiens non linéaires dans le plan. C.R. Dokl. Acad. Sci. 297, 307-310 (1983)

17. Brezis, H., Lieb, E.: Minimum action solutions of some vector field equations. Commun. Math. Phys. 96, 97-113 (1984)

18. Pecher, H.: Low-energy scattering for nonlinear Klein-Gordon equations. preprint

Communicated by A. Jaffe

Received November 19, 1984 